\title{
Micorwave observations of the Chinese Solar Broadband Radio Spectrometer at Huairou
}

\author{
Chengming Tan ${ }^{1,2}$, Baolin Tan ${ }^{1}$, Yihua Yan ${ }^{1}$, and Yuying Liu ${ }^{1}$ \\ ${ }^{1}$ Key Laboratory of Solar Activity, National Astronomical Observatories, Chinese Academy of \\ Science, Beijing 100012 \\ ${ }^{2}$ Sate Key Laboratory of Space Weather, Chinese Academy of Sciences, Beijing 100190 \\ email: tanchm@nao.cas.cn
}

\begin{abstract}
This work presents an overview of the microwave observations of the Chinese Solar Broadband Radio Spectrometer at Huairou (SBRS/Huairou) during 1997-2011. The relationships between the microwave bursts and solar flares and the calibration of spectrometers are also studied.
\end{abstract}

Keywords. radio bursts, solar flare, calibration

\section{Observations and Calibration}

The Chinese Solar Broadband Radio Spectrometer at Huairou (SBRS/Huairou) contains three frequency bands: 1.0-2.0 GHz, 2.6-3.8 GHz, 5.2-7.6 GHz (Fu et al. (2004)). Since 1997, it has observed thousands of microwave bursts and accumulated a great number of observation data. Table 1 presents a list of the radio bursts and the related X-class flares. There are 54 X-class flares observed by SBRS/Huairou during 1997-2011. As for all class flares, 2661 soft X-ray flares (including 17 X-class, 215 M-calss, 2429 C-calss, and 166 B-calss flares) were recorded from National Geophysical Data Center (NGDC) in 2000. Among them, 1224 flares ( 7 X-calss, 98 M-calss, 79 C-class, and 71 B-class flares) were observed by SBRS/Huairou. Besides these events, 77 microwave bursts without obviously related soft X-ray flares were also observed by SBRS/Huairou in the same year. They took place tens of minutes to serveral hours before or after some flares. Table 2 presents the relationship between microwave bursts and solar flares observed in 2000 .

Following the fundamental method of Tanaka et al. (1973) and Yan et al. (2002), we make the analysis of the calibration constants and the standard deviation of calibration at three frequency bands of SBRS during 1997-2009. At 2.6-3.8 GHz band, Tan et al. (2009) indicated that the calibration result has correlation with the local air temperature. A correction factor that varied with local air temperature was considered. This correction can reduce the error of calibration about $30 \%$. We choose the daily noon solar flux without sunspots before or after three days as the radio flux of quiet sun, and obtain the spectrum of quiet solar radio emission. Fig. 1 shows the comparison between the observation and numerical simulation (Smerd (1950)) of quiet solar radio emission. It indicated the electron temperature of chromospheres about $\sim 10^{4} \mathrm{~K}$.

\section{Summary}

From our analysis on the observations of SBRS/Huairou, we may obtain the following conclusions: all the powerful flares with class $\geqslant M 4.0$ were observed to have relationships with microwave bursts, part of the other flares are also associated with the microwave bursts. The calibration analysis indicates that the calibration constants are correlated 
Table 1. Microwave bursts and the recorded X-Class soft X-ray flares observed by SBRS

\begin{tabular}{|c|c|c|c|c|c|c|c|c|c|c|c|c|c|c|c|c|}
\hline |Year & | 1997 & | 1998 & | 1999 & $\mid 2000$ & | 2001 & $\mid 2002$ & 2003 & | 2004 & | 2005 & $\mid 2006$ & $\mid 2007$ & $7 \mid 2008$ & $8 \mid 200$ & $9 \mid 2010$ & $0 \mid 2011$ & | Tot. | \\
\hline Radio burst & 31 & $\mid 181$ & 113 & $\mid 324$ & | 631 & 531 & 369 & 240 & 171 & 31 & 15 & 0 & 0 & 15 & | 147 & | $2790 \mid$ \\
\hline \begin{tabular}{|l} 
associated \\
X-Class flares
\end{tabular} & 1 & 8 & 1 & 7 & 5 & 5 & 7 & 5 & 11 & 1 & 0 & 0 & 0 & 0 & 3 & 54 \\
\hline X-Class flares & 3 & 14 & 4 & 17 & 21 & 11 & 20 & 12 & 18 & 4 & 0 & 0 & 0 & 0 & 8 & 132 \\
\hline
\end{tabular}

Table 2. The relationship between microwave bursts and soft X-ray flares in 2000

\begin{tabular}{|c|c|c|c|c|c|c|c|c|c|}
\hline All SXR flares (2661) & | 17 X-class & 215 & 5 M-Class & 2429 & 9 C-Class & 166 & B-Class & s $\mid$ no & o flare \\
\hline SXR flares with observation of SBRS & 7 & 1 & 98 & I & 1048 & 1 & 71 & 1 & - \\
\hline Associated radio burst & 7 & 79 & $(\sim 80 \%)$ & I & 160 & I & 1 & I & 77 \\
\hline
\end{tabular}

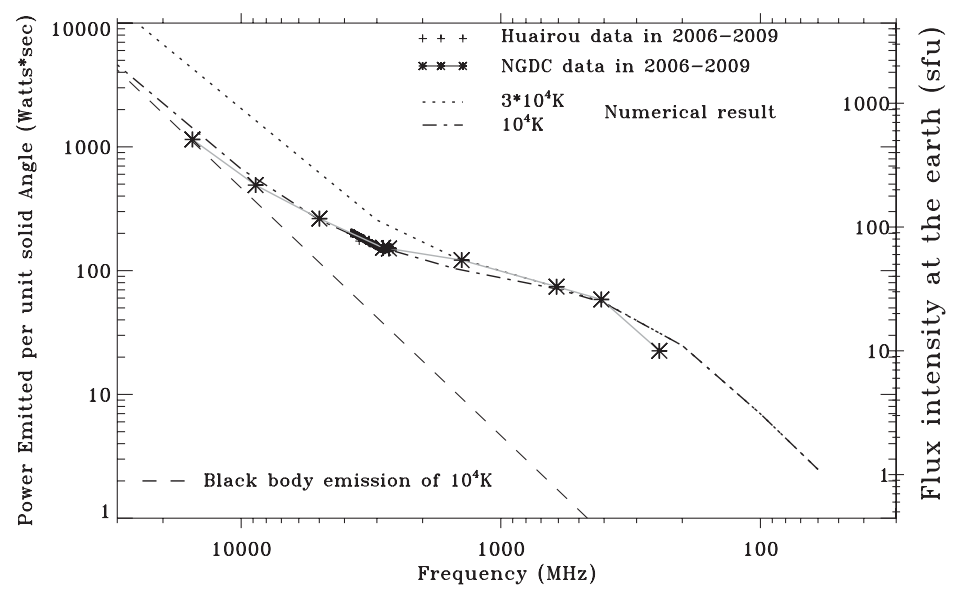

Figure 1. Comparison between observations and numerical result of the spectrum of quiet solar radio emission

with the local air temperature. One possible reason is that the electronic apparatus are affected by the air temperature.

\section{Acknowledgements}

This work was supported by the NSFC (11103044, 11273030, 11263004, 10921303), MOST2011CB811401, and open foundation of Sate Key Laboratory of Space Weather, CAS. The online data of NGDC were used.

\section{References}

Fu Q. J., Ji H. R., Qin, Z. H., et al. 2004, Sol Phys., 222, 167

Smerd, S. F. 1950, AuSRA, 3, 34

Tanaka, H., Castelli, J. P., Covington, A. E., et al. 1973, Solar Phys., 29, 243

Tan, C. M., Yan, Y. H., Tan, B. L., \& Xu, G. L. 2009, Sci China Ser G, 52, 1760

Yan, Y. H., Tan, C. M., Xu, L., et al. 2002, Sci China Ser A, 45 Supp: 89 